\title{
Perspectives of Chronic Disease Management Among Persons with HIV: A Qualitative Study
}

This article was published in the following Dove Press journal:

Patient Preference and Adherence

\author{
Laura Mkumba' \\ Charles Muiruri $\mathbb{D}^{2}$ \\ Keva Garg ${ }^{3}$ \\ Melissa $\mathrm{H}$ Watt $^{4}$ \\ Nwora Lance Okeke ${ }^{5}$ \\ 'FHI360, Durham, NC, USA; \\ ${ }^{2}$ Department of Population Health \\ Sciences, Duke University, Durham, NC, \\ USA; ${ }^{3}$ Department of Medicine, Johns \\ Hopkins University School of Medicine, \\ Baltimore, MD, USA; ${ }^{4}$ Department of \\ Population Health Sciences, University of \\ Utah, Salt Lake City, UT, USA; ${ }^{5}$ Division \\ of Infectious Diseases, Duke University \\ Medical Center, Durham, NC, USA
}

Correspondence: Nwora Lance Okeke Duke University Medical Center, 315

Trent Drive, Rm. 178, Durham, NC

27707, USA

Tel $+\mid 9196842579$

$\mathrm{Fax}+19196817494$

Email lance.okeke@duke.edu
Introduction: Persons with HIV (PWH) are living to advanced age as a result of ART. These epidemiological changes highlight the importance of innovating chronic care delivery of PWH, but there is limited research regarding patient preferences for chronic care delivery. Methods: We conducted in-depth interviews of $20 \mathrm{PWH}$ who receive care at the Duke Infectious Diseases Clinic. Manuscript was coded and we used thematic analysis to identify emerging themes from interviewees' responses.

Results: Insights of the interviews revealed a strong affinity of PWH with their HIV providers and a reliance on them for primary care as a result. Participants also expressed a strong preference for receiving NCD care from a single provider, regardless of their current chronic disease care configuration. Participants also stated a willingness to embrace new roles of non-provider HIV clinic staff in their chronic disease care.

Conclusion: Overall, persons living with HIV prefer consolidation and co-location of their care, and are willing to endure minor inconveniences to accommodate this preference. Efforts towards promoting primary care integration into HIV clinics are warranted.

Keywords: chronic disease management, primary care, non-communicable diseases, patient perspectives

\section{Introduction}

With over 21 million on effective antiretroviral therapy (ART), people with HIV (PWH) are living longer than ever before. ${ }^{1}$ As a result, non-communicable chronic diseases (NCD) have become increasingly prevalent in this population. ${ }^{2}$ Due to a number of factors, $\mathrm{PWH}$ are at increased risk of many chronic NCDs. ${ }^{3-5}$ Furthermore, certain antiretrovirals place PWH at increased risk of chronic NCDs like dyslipidemia and impaired glucose tolerance. ${ }^{6,7}$ As a result, recent efforts have focused on understanding chronic NCD epidemiology and healthcare delivery among PWH in the United States.

With the changing burden of disease among PWH, the need to re-evaluate healthcare delivery to this population is essential. ${ }^{8}$ Debates exist regarding the ideal care configuration to render NCD care to this population. HIV providers have expressed discomfort with taking on the additional responsibilities of managing chronic NCDs. ${ }^{9}$ This has led to a call for additional training for HIV providers to manage NCDs, as well as better coordination with relevant providers. ${ }^{8,10}$ While previous studies have explored patients' preferences for primary care, few studies have explored patients' perspectives on consolidated care (all HIV and non-HIV care provided by a single provider) vs a shared care, two-provider model. ${ }^{11}$ The 
goals of this study were to understand how PWH perceive their NCD care, to assess patients' preferences for provider models, and to document patients' suggestions for how NCD care delivery can be improved upon.

\section{Methods}

\section{Setting}

The study was conducted at the Duke Adult Infectious Diseases (ID) Clinic. Located in Durham, North Carolina, the Duke ID Clinic provides medical care to approximately $1900 \mathrm{PWH}$. HIV care is rendered by 17 IDtrained faculty, 2 physician assistants, and 7 infectious diseases fellows. All providers are in the clinic once a week for a half-day session, usually with $10-15$ patients scheduled in each session. In $2017,48 \%$ of HIV clinic patients received chronic NCD care outside of the clinic.

\section{Sample and Recruitment}

Clinic patients age $\geq 40$ years, in care at Duke for $\geq 2$ years and on stable ART for $\geq 1$ year, and with $\geq$ chronic NCD were eligible to participate in the study. Eligible chronic NCDs were hypertension, diabetes, arthritis (any type), hyperlipidemia, coronary artery disease, congestive heart failure, asthma, COPD, osteoporosis, osteopenia, chronic kidney disease and thyroid dysfunction.

We sought to recruit an even number of patients with and without alternate primary care providers (PCPs) to increase diversity of perspectives. Patients were recruited via study fliers in the clinic and advertising at community advisory board meetings. In addition, we reviewed electronic health records to identify potentially eligible patients and approached them during clinic encounters. Participants were enrolled between February 2016 and October 2017. In accordance with current scientific transparency standards, de-identified transcripts from our study are available upon request.

\section{Procedures}

Participants provided written informed consent prior to study enrollment. The participants' consent included publication of anonymized responses, and that the study was conducted in accordance with the Declaration of Helsinki. Participants completed a single semistructured in-depth interview with a study team member formally trained in qualitative interviewing for research purposes. Topics covered in the interview guide include: experience with HIV provider, experience with primary care provider, preferences of chronic care delivery, and acceptability of HIV clinic support staff involvement in NCD care. Interviews were conducted in a private room in the ID clinic, and participants were compensated for their time. Audio recordings of the interviews were transcribed verbatim, and transcripts were reviewed by the team for accuracy. All transcripts were de-identified. This study was approved by the Duke University Health System Institutional Review Board.

\section{Analysis}

Inductive thematic analysis was used to identify themes related to our research questions. ${ }^{12}$ First, two members of the study team read the transcripts and wrote narrative memos that summarized and organized each transcript. The memos were organized by section headings of the interviews, and direct quotes were integrated to illuminate core ideas and support the development of codes. The narrative memos and transcript pairs were reviewed together by at least two team members to ensure that they were comprehensive. The codebook was developed using a priori codes based on the research questions and supplemented by data-driven codes from transcripts and memos. Two members of the study team coded the interviews independently in NVivo (version 10). Queries were run in NVivo to collate coded text. After agreeing on common themes, analytic memos were written to synthesize the data for the themes across study participants. Notable quotations were highlighted as textual evidence and to provide context to the themes.

\section{Results}

Overall, 22 participants were enrolled in the study. One participant gave consent but did not attend the scheduled interview. Another patient did not meet inclusion criteria after informed consent was completed. There were 20 patients in the final analysis cohort (Table 1). Among interviewed patients, $13(65 \%)$ were Black, 19 (95\%) were non-Hispanic, and $6(30 \%)$ identified as women. The median age for participants was 52.5 [interquartile range (IQR) 47.5-56.5, Range 44-67]. Median time from diagnosis of HIV was 18 years [IQR 11-23.5 years]. Overall, 12 out of $20(60 \%)$ participants had an alternate PCP in addition to a HIV provider. Four main themes emerged from the interviews: primacy of the relationship with HIV provider, variable experiences with non-HIV PCPs, preference for an integrated care delivery model, and receptiveness to involvement of HIV support staff in NCD care. 
Table I Participant Characteristics $(\mathrm{n}=20)$

\begin{tabular}{|l|l|}
\hline Female & $6(30)$ \\
\hline Race & $1093(66)$ \\
\hline Black & $13(65)$ \\
\hline White & $7(35)$ \\
\hline Other & 0 \\
\hline Median age [IQR] & $52.5[47.5,56.5]$ \\
\hline Median years known to be HIV positive & $18[11,23.5]$ \\
\hline Median years at Duke HIV Clinic & $10[6,12]$ \\
\hline Hypertension & $14(70)$ \\
\hline Hyperlipidemia & $7(35)$ \\
\hline Diabetes & $3(15)$ \\
\hline Asthma/COPD & $4(20)$ \\
\hline Osteoarthritis & $3(15)$ \\
\hline
\end{tabular}

Abbreviations: COPD, chronic obstructive pulmonary disease; IQR, interquartile range.

\section{Primacy of Relationship with HIV Providers}

All participants expressed satisfaction with care received from their provider. Participants were comfortable asking their providers questions and were satisfied with provider feedback overall. Many participants described loyalty to their HIV providers for their central role in providing care during the worst periods of their HIV infection. Many participants also assigned positive personal attributes to their HIV provider.

Extremely satisfied. I credit him with really saving my life ....I feel fortunate to have found him. I feel like this is the best fit for me. His manner, you can tell he's concerned. He's really involved with his patients on another level. You don't have that relationship and that dialogue with a lot of doctors because a lot of doctors are distracted, distant - Participant 02

It's not like any doctor. When I ask somebody about their doctor, my relationship with my doctor is totally different. Alright. But he really, really been looking out for me the last six years. So far, I wouldn't trade him for the world. Participant 17

Among participants who received NCD care from their HIV provider, the most cited reasons for consolidated care were healthcare cost and comfort with their HIV provider.
Other participants expressed comfort with their care configuration and were not keen on starting a new therapeutic relationship with other providers. As Participant 22 noted:

[The HIV clinic] knows my history, they know my charts, everything's here ... 'Cause I know who to contact if something's wrong. I don't have to go through my phone and try to dig out another name and number. I can call the clinic and say, hey, this is what's going on. Can you get me in?

Many patients also expressed a perceived reduction in quality of care sought outside of the HIV clinic.

I grew very accustomed to having the best of the best taking all the care of me, not sending me to a different doctor to manage a cold or a sneeze or a sniffle or something like that. My doctor did everything for me. I made the personal choice to stay here and keep all my information kind of together instead of having to have too many doctors communicate with each other because that can create problems.- Participant 20

The primary caveat expressed by some patients about receiving all care from their HIV provider was accessibility related to scheduling issues with a once-weekly specialty clinic.

Overall pretty satisfied. The only thing is, a lot of times I have to reschedule because he has to cancel, and that's one concern I have. One year we had to reschedule every appointment I had, and I wasn't very happy with that. It is kind of stressful because, that means I have to get off another time from work, he's only available certain days, it's Thursday and that's really a busy time for me at work. If I have to reschedule it just throws things awry sometimes. - Participant 09

However, none of the patient perceived this as a deterrent to continuing care at the HIV clinic.

\section{Experience with Non-HIV Primary Care Providers}

Overall, participants had varied responses about care with alternate PCPs. Most participants stated that they only attended brief annual checkups with these providers. Almost universally, participants expressed a lack of depth of relationship with their PCP compared to the relationship with their HIV providers. Many patients cited administrative requirements related to insurance coverage as the reason why they saw a PCP at all. A few participants 
stated that their HIV providers had instructed them to seek out a PCP for their NCD care.

I will tell you one thing, when I first started care I assumed that the HIV doctor would be a little bit more of the main care provider since so many things track back to it [HIV]. I guess I was a little disappointed to learn he's very much strictly tied to my HIV treatment and that's about it ... He made it very clear right away I'm not going to be your primary care physician. - Participant 7

Despite having alternate providers, many participants reported frequently validating medical advice received from other providers with their HIV provider. This input from HIV providers was most frequently sought when new medications were prescribed by PCPs. As Participant 19 states:

So anytime I deal with another doctor for anything, I don't really understand it until I come see [my HIV Provider] and then he breaks it down and helps me to understand it fully ... So I may see the other people but until I get [my HIV Provider's] opinion, their opinion, you know. He has to verify it for me.

\section{Preference for Integrated Chronic Care Delivery Model}

In general, participants preferred an integrated care model where all of their care was consolidated in one place, with one provider. Participant 3 reports: "I wish my HIV doctor could provide everything ... If I could get all my care in one place that would be wonderful rather than travelling to different places". A few interviewees however considered a multi-provider team-based approach with the HIV provider as the team lead:

It would actually be a team of doctors, if they sat down and met once a month to see what my progress is ... "She's done this, she's did that, and now she doesn't have to take the blood pressure medicine" ... but still the main focus is still keeping my HIV doctor, who would be the head of my team, regardless of who else was on there. - Participant 13

Among participants with an HIV provider and a PCP, most believed that there was passive communication between providers, primarily through chart review. In general, they found this method of communication acceptable. Participant 8 explained:

When I come in, the doctors are knowledgeable about what the other doctors have done, whether they looked at it that morning or the night before ... but I know they're knowledgeable of what's going on at the other doctors.

In general, patients expressed that communication between their providers is valuable and can have a positive impact on their overall health outcomes.

I think it [care coordination] affects [health outcomes]. As long as they're communicating, even with my neurologist, I think as long as they're all talking to one another it benefits me. At least everybody is on the same page. Participant 6

A few patients expressed dissatisfaction with a lack of communication between providers and expressed concern that poor communication would impact their health outcomes:

Last year I had a situation ... I had been on medication for high blood pressure with my HIV provider and another medication for Bell's palsy, gabapentin and I had to go to my primary doctor, and he took me off the medication because they were making my heart rate too slow. So therefore, my doctors should have interacted then [to catch the slow heartrate], but they didn't .... Had there been communication, my HIV provider would have caught it earlier - Participant 3

\section{Increased Involvement of HIV Clinic Support Staff}

Overall, patients reported minimal interaction with HIV clinic support staff (nurses, social workers, pharmacists). However, most were comfortable with the support staff being more involved with their care. Many patients acknowledged the value of an expanded social workers that would include care coordination across providers:

... At the very least, more social work needs to be part of everybody's care, at least on a yearly basis. Secondarily, if they could actually expand that role and act as an ombudsman for making sure that everybody has a team of people for their care and managing that that's being followed through and that sort of stuff - Participant 7

Despite some expressed skepticism from participants about the role of nurses and pharmacists in their care, some suggested that perhaps the nurses could assist in improving health literacy. Participant 17 explained:

I talk to Dr. X about, I don't get the full understanding about it, I can go ask one of the nurses. She'll break it right down to me like that ... I can relate to them more ... 
In general, patients were open and appreciative of the overall idea of having the clinic support staff become more invested in their well-being. For many participants, a phone call from clinic support staff as added communication was a welcome consideration.

I think it could. I think it would give a sense of wellbeing and everything because a lot of times you can feel maybe abandoned. It helps to let you know that you're not alone. That someone is there, someone cares. - Participant 02

\section{Discussion}

Our study examined PWH perceptions of NCD care in the ambulatory care setting. Participants were very satisfied with NCD care received from their HIV providers. While participants were generally satisfied with NCD care received from their PCP, patient-provider relationships were not as strong as with HIV providers. Participants generally valued inter-provider communication but generally perceived inadequacies in communication between their providers. Participants reported minimal interaction with the HIV clinic support staff, but were comfortable with their increased participation in their care.

A prominent feature of participants' relationship with HIV providers was a perceived sense of loyalty, generally absent in their relationship with PCPs. This dynamic is likely the result of a unique role that HIV providers play in their overall health. Given the unique dynamic of the initiation of life-saving and rapidly health-restoring ART, generally positive participant attributions to their HIV providers are not surprising. ${ }^{13}$ Some patients stated that they had a PCP only to meet insurance requirements, but they still viewed their HIV providers as their primary providers. These findings are consistent with prior studies, and provide a considerable argument for transitioning toward integrated care for persons living with HIV. ${ }^{11}$ Most published experience with integration of NCD care into the HIV clinic setting has come from sub-saharan Africa, and in general has proven effective in reducing the burden of NCD-related comorbidity in that population $^{14}$. Ryan White Part C-funded HIV clinics around the country have effectively integrated NCD management into the HIV clinic setting for several years. ${ }^{15}$ Unfortunately, the best model for clinics without this funding remain elusive. ${ }^{10}$ Interestingly, despite the wellestablished link between HIV-associated stigma and medical mistrust, our participants did not explicitly mention perceived stigma as a barrier to healthcare access. ${ }^{16,17}$
All patients expressed the need for care coordination between their providers. This need was primarily driven by a desire for "peace of mind", and perceived such communication as suggestive of a superior quality of care overall. Patients also saw social workers and case managers as potential bridges to their providers through interim phone calls. These results point towards innovating service delivery models for HIV patients living with chronic non-HIV conditions. Several options that have been explored with varying success. ${ }^{10,18,19}$ Given the receptiveness to additional involvement by the clinical support staff, many opportunities arise for the development of innovative models to improve NCD delivery in this context. This multidisciplinary approach could lessen the burden of HIV providers while ensuring that patients are receiving comprehensive NCD care.

There are many implications of these results. First, the lack of value placed on relationships with PCPs could imply that patients may not be appropriately aware of the role PCPs in their healthcare. This demonstrates a need for more educational interventions targeted towards PWH on multimorbidity of HIV and non-HIV chronic conditions, as well as the roles that different members of the health system can play in helping them manage these conditions. Second, many participants expressed concern about accessibility to their HIV providers. While patients also expressed the desire of integrating services, issues of accessibility would likely not improve if their HIV provider were to take on the additional burden of managing their patients' non-HIV conditions. Given that longer consultation time with providers and decreased waiting times are key components in patient satisfaction, it is also unlikely that satisfaction with the care they receive would improve. ${ }^{20}$ Finally, patients who rely on HIV providers to manage their non-HIV chronic conditions may receive sub-optimal NCD care overall. ${ }^{21-23}$ However, given participants' expressed loyalty to their HIV provider, efforts may be best served focusing on how to improve NCD care rendered by HIV providers, as opposed to taskshifting.

The study findings must be interpreted in light of the limitations. Our sampling strategy was reliant on recruiting patients while they were in the clinic for their doctor's appointments. While most of the patients who were we approached were open to participation, it is possible that we may have missed a population who could have provided additional insights into the study goals. Our inclusion criteria on clinic attendance excluded the perspectives of individuals who struggled with care engagement. 
Initially, we sought to recruit a sample stratified by years living with HIV diagnosis. However, we struggled to recruit participants who were recently diagnosed $(<5$ years) and our sample consisted primarily of PWH for over 10 years. Missing individuals with relatively new diagnoses could have skewed our findings and precluded us from making comparisons by time since diagnosis.

\section{Conclusion}

Multimorbidity of non-HIV NCD in PWH has highlighted the importance of improving NCD care in this population. In a study population of HIV-infected individuals who were diagnosed with other non-HIV chronic conditions, participants preferred comprehensive and integrated care that manages their HIV and NCD. Participants either wanted all their care to be provided by their HIV provider or a team of providers with their HIV provider as the "head provider." Participants' willingness to having a member of the clinic support staff being more involved in the care presents a new avenue of incorporating multidisciplinary care team to improve the NCD health outcomes for PWH.

\section{Acknowledgments}

We thank the patients and staff of the Duke Adult Infectious Diseases Clinic for their contribution and support throughout this project.

\section{Funding}

This work was supported by the National Heart, Lung and Blood Institute (K23-HL137611) and the Duke Center for AIDS Research (P30 AI064518). CM was supported by Diversity Supplements from the National Heart, Lung, and Blood Institute (U01HL142099-01S) and National Institute of Minority Health and Development (R01 MD013493-01).

\section{Disclosure}

The authors report no conflicts of interest in this work.

\section{References}

1. WHO. Antiretroviral therapy (ART) coverage among all age groups (Internet). WHO; [cited June 12, 2019]. Available from: http://www. who.int/gho/hiv/epidemic_response/ART_text/en/. Accessed December $16,2020$.

2. Gallant J, Hsue PY, Shreay S, Meyer N. Comorbidities among US patients with prevalent HIV infection-a trend analysis. $J$ Infect Dis. 2017;216(12):1525-1533. doi:10.1093/infdis/jix518
3. Hsue PY, Deeks SG, Hunt PW. Immunologic basis of cardiovascular disease in HIV-infected adults. J Infect Dis. 2012;205(Suppl 3): S375-S382. doi:10.1093/infdis/jis200

4. Schouten J, Wit FW, Stolte IG, Kootstra NA, van der Valk M, Geerlings SE; AGEhIV Cohort Study Group. Cross-sectional comparison of the prevalence of age-associated comorbidities and their risk factors between HIV-infected and uninfected individuals: the AGEhIV cohort study. Clin Infect Dis. 2014;59(12):1787-1797. doi:10.1093/cid/ciu701

5. Guaraldi G, Orlando G, Zona S, et al. Premature age-related comorbidities among HIV-infected persons compared with the general population. Clin Infect Dis. 2011;53(11):1120-1126. doi:10.1093/cid/cir627

6. van Zoest RA, Wit FW, Kooij KW, et al. Higher prevalence of hypertension in HIV-1-infected patients on combination antiretroviral therapy is associated with changes in body composition and prior stavudine exposure. Clin Infect Dis. 2016;63(2):205-213. doi:10.1093/cid/ciw285

7. Worm SW, Sabin C, Weber R, et al. Risk of myocardial infarction in patients with HIV infection exposed to specific individual antiretroviral drugs from the 3 major drug classes: the data collection on adverse events of anti-HIV drugs (D:A:D) study. J Infect Dis. 2010;201(3):318-330. doi:10.1086/649897

8. Chu C, Selwyn PA. An epidemic in evolution: the need for new models of HIV care in the chronic disease era. J Urban Health. 2011;88(3):556-566. doi:10.1007/s11524-011-9552-y

9. Fultz SL, Goulet JL, Weissman S, et al. Differences between infectious diseases - certified physicians and general medicine-certified physicians in the level of comfort with providing primary care to patients. Clin Infect Dis. 2005;41(5):738-743. doi:10.1086/432621

10. Lakshmi S, Beekmann SE, Polgreen PM, Rodriguez A, Alcaide ML. HIV primary care by the infectious disease physician in the United States - extending the continuum of care. AIDS Care. 2018;30 (5):569-577. doi:10.1080/09540121.2017.1385720

11. Cheng QJ, Engelage EM, Grogan TR, Currier JS, Hoffman RM. Who provides primary care? An assessment of HIV patient and provider practices and preferences. J AIDS Clin Res. 2014;5(11). doi:10.4172/ 2155-6113.1000366

12. Guest G, MacQueen K, Namey E Applied thematic analysis (Internet). 2455 Teller Road, Thousand Oaks California 91320 United States: SAGE Publications, Inc.; 2012 Available from: http://methods.sagepub.com/book/applied-thematic-analysis. Accessed December 16, 2020.

13. Brion J. The patient-provider relationship as experienced by a diverse sample of highly adherent HIV-infected people. J Assoc Nurses AIDS Care. 2014;25(2):123-134. doi:10.1016/j.jana.2013.01.006

14. Njuguna B, Vorkoper S, Patel P, et al. Models of integration of HIV and noncommunicable disease care in sub-Saharan Africa: lessons learned and evidence gaps. AIDS. 2018;32 Suppl 1(Suppl 1):S33S42. doi:10.1097/QAD.0000000000001887

15. Gallant JE, Adimora AA, Carmichael JK, Horberg M, Kitahata M; Quinlivan EB Ryan White Medical Providers, C. Essential components of effective HIV care: a policy paper of the HIV Medicine Association of the Infectious Diseases Society of America and the Ryan White Medical Providers Coalition. Clin Infect Dis. 2011;53 (11):1043-1050. doi:10.1093/cid/cir689

16. Stockton MA, Giger K, Nyblade L. A scoping review of the role of HIV-related stigma and discrimination in noncommunicable disease care. PLoS One. 2018;13(6):e0199602. doi:10.1371/journal.pone.019 9602

17. Ion A, Elston D. Examining the health care experiences of women living with human immunodeficiency virus (HIV) and perceived HIV-related stigma. Women's Health Issues. 2015;25(4):410-419. doi:10.1016/j.whi.2015.03.012

18. Beane SN, Culyba RJ, DeMayo M, Armstrong W. Exploring the Medical Home in Ryan White HIV care settings: a pilot study. J Assoc Nurses AIDS Care. 2014;25(3):191-202. doi:10.1016/j. jana.2013.10.007 
19. Greene ML, Tan JY, Weiser SD, et al. Patient and provider perceptions of a comprehensive care program for HIV-positive adults over 50 years of age: the formation of the Golden Compass HIV and aging care program in San Francisco. PLoS One. 2018;13(12):e0208486e0208486. doi:10.1371/journal.pone.0208486

20. Asghari S, Hurd J, Marshall Z, et al. Challenges with access to healthcare from the perspective of patients living with HIV: a scoping review \& framework synthesis. AIDS Care. 2018;30 (8):963-972. doi:10.1080/09540121.2018.1435848

21. Okeke NL, Chin T, Clement M, Chow S-C, Hicks CB. Coronary artery disease risk reduction in HIV-infected persons: a comparative analysis. AIDS Care. 2016;28(4):475-482. doi:10.1080/09540121.2015.1099602
22. Ladapo JA, Richards AK, DeWitt CM, et al. Disparities in the quality of cardiovascular care between HIV-infected versus HIV-uninfected adults in the United States: a cross-sectional study. J Am Heart Assoc. 2017;6(11):e007107. doi:10.1161/JAHA.117.007107

23. Burkholder GA, Tamhane AR, Salinas JL, et al. Underutilization of aspirin for primary prevention of cardiovascular disease among HIV-infected patients. Clin Infect Dis. 2012;55(11):1550-1557. doi:10.1093/cid/cis752

\section{Publish your work in this journal}

Patient Preference and Adherence is an international, peer-reviewed, open access journal that focusing on the growing importance of patient preference and adherence throughout the therapeutic continuum. Patient satisfaction, acceptability, quality of life, compliance, persistence and their role in developing new therapeutic modalities and compounds to optimize clinical outcomes for existing disease states are major areas of interest for the journal. This journal has been accepted for indexing on PubMed Central. The manuscript management system is completely online and includes a very quick and fair peer-review system, which is all easy to use. Visit http:// www.dovepress.com/testimonials.php to read real quotes from published authors. 